\title{
Pairwise Distance Matrix Computation for Multiple Sequence Alignment on the Cell Broadband Engine
}

\author{
Adrianto Wirawan, Bertil Schmidt, and Chee Keong Kwoh \\ School of Computer Engineering, Nanyang Technological University, Singapore 639798 \\ \{adri0004, asbschmidt, asckkwoh\}@ntu.edu.sg
}

\begin{abstract}
Multiple sequence alignment is an important tool in bioinformatics. Although efficient heuristic algorithms exist for this problem, the exponential growth of biological data demands an even higher throughput. The recent emergence of accelerator technologies has made it possible to achieve a highly improved execution time for many bioinformatics applications compared to general-purpose platforms. In this paper, we demonstrate how the PlayStation ${ }^{\circledR} 3$, powered by the Cell Broadband Engine, can be used as a computational platform to accelerate the distance matrix computation utilized in multiple sequence alignment algorithms.
\end{abstract}

Keywords: multiple sequence alignment, cell broadband engine.

\section{Introduction}

Multiple sequence alignment (MSA) of many nucleotides or amino acids is an important tool in bioinformatics. It can identify patterns or motifs to characterize protein families, and is therefore utilized to detect homology between sequences as well as to perform phylogenetic analysis. Many MSA heuristics have been proposed to reduce the exponential complexity of computing optimal MSAs. Heuristic MSA implementations include MSA[1], ClustalW[2], T-Coffee[3], MAFFT[4], DIALIGN P[5] and PRALINE[6]. ClustalW[2] has over 26,000 citations in the ISI Web of Science and is considered to be one of the most popular MSA tools. It is based on the progressive alignment method. Although not optimal, this method can produce reasonably good alignments at a good efficiency. However, the exponential growth of biological data demands an even better throughput. Thus, software approaches to improve the performance of ClustalW have been introduced, including caching[8, 9] and parallel processing[10-12].

The recent emergence of accelerator technologies such as FPGAs, GPUs and specialized processors have made it possible to achieve an improvement in execution time for many bioinformatics applications compared to current general-purpose platforms at a low cost. Recent usage of easily accessible accelerator technologies to improve the ClustalW algorithm include FPGA[13] and GPU[14].

Our profiling of ClustalW has revealed that distance matrix computation is the most time consuming stage and typically takes up more than $90 \%$ of the overall runtime. 
Therefore, accelerating this phase would greatly improve the performance as a whole. In this paper, we investigate how the Cell Broadband Engine, a heterogeneous multi-core architecture, can be used as a computational platform to accelerate the distance matrix computation in ClustalW. By taking advantage of multiple processors as well as SIMD vectorization, we are able to achieve speedups of two orders of magnitude compared to the publicly available sequential ClustalW implementation.

The rest of this paper is organized as follows. Section 2 highlights important features of the Cell Broadband Engine. Section 3 briefly describes the ClustalW algorithm. Section 4 presents our mapping of the distance matrix computation onto the Cell Broadband Engine. Experimental results are presented in Section 5. Section 6 concludes the paper.

\section{Cell Broadband Engine}

The Cell Broadband Engine[15] (Cell BE) is a recently introduced single-chip heterogeneous multi-core processor, which is developed by Sony, Toshiba and IBM. The Cell BE offers a unique assembly of thread-level and data-level parallelization options. It is operating at the upper range of existing processor frequencies $(3.2 \mathrm{GHz}$ for current models). Several examples of bioinformatics applications that has been ported to the Cell BE architecture include Folding@Home[16], FASTA[17], SmithWaterman[18] and RAxML[19].

The Cell BE combines an IBM PowerPC Processor Element (PPE) and eight Synergistic Processor Elements (SPEs)[20]. An integrated high-bandwidth bus called the Element Interconnect Bus (EIB) connects the processors and their ports to external memory and I/O devices. The block diagram of the Cell BE architecture is shown in Figure 1.

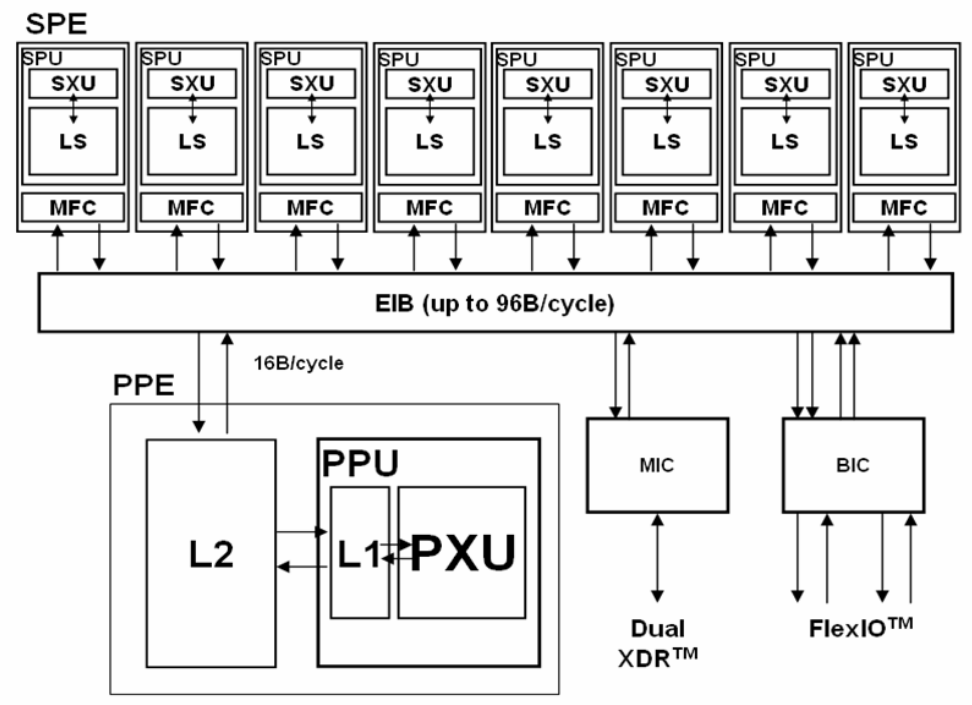

Fig. 1. Block diagram of the Cell Broadband Engine Architecture 
The PPE is a 64-bit Power Architecture core and contains a 64-bit general purpose register set (GPR), a 64-bit floating point register set (FPR), and a 128-bit Altivec register set. It is fully compliant with the 64-bit Power Architecture specification and can run 32-bit and 64-bit operating systems and applications. Each SPE is able to run its own individual application programs. Each SPE consists of a processor designed for streaming workloads, a local memory, and a globally coherent Direct Memory Access (DMA) engine. The EIB is a 4-ring structure, and can transmit 96 bytes per cycle, for a bandwidth of 204.8 Gigabytes/second. The EIB can support more than 100 outstanding DMA requests.

The most distinguishing feature of the Cell BE lies within the variety of the processors it has, i.e. the PPE and the SPEs. Heterogeneous multi-core systems can lead to decreased performance if both the operating system and application are unaware of the heterogeneity. The PPE is designed to run the operating system and, in many cases, the top-level control thread of an application, while the SPEs is optimized for compute intensive applications, hence, providing the bulk of the application performance.

The SPE can access RAM through direct memory access (DMA) requests. The DMA transfers are handled by the Memory Flow Controller (MFC). The MFC provides the interface, by means of the EIB, between the local storage of the SPE and main memory. The MFC supports DMA transfers as well as mailbox and signalnotification messaging between the SPE and the PPE and other devices. Data transferred between local storage and main memory must be 128-bit aligned. The size of each DMA transfer can be at most $16 \mathrm{~KB}$. DMA-lists can be used for transferring large amounts of data (more than $16 \mathrm{~KB}$ ). A list can have up to 2,048 DMA requests, each for up to $16 \mathrm{~KB}$.

The PS3 uses the Cell Broadband Engine as its CPU, hence making it possible for users to create a high-powered computing environment for a fraction of the cost of a Cell Blade server. The PS3 utilizes seven of the eight SPEs, in which the eighth SPE is disabled to improve chip yields, i.e. chips do not have to be discarded if one of the SPEs is defective. Only six of the seven SPEs are accessible to developers as one is reserved by the operating system. Generally available PS3's can be used for scientific high performance computing through installation of Linux (e.g. Fedora Core or Yellow Dog). Programs can be developed the using freely available C-based Cell BE SDK[21].

\section{Multiple Sequence Alignment}

ClustalW is highly popular sequential MSA software. It implements a progressive alignment method[22], i.e. it adds sequences one by one to the existing alignment to build a new alignment. The order of sequences to be added to the new alignment is indicated by a phylogenetic tree, which is called a guide tree. The guide tree is constructed from the similarity of all possible pairs of sequences stored in the distance matrix. Overall, the three stages of the ClustalW algorithm can be summarized as follows: 
1. Distance matrix computation: each pair of sequences is aligned separately to calculate a respective distance value. These values are stored in a so-called distance matrix.

2. Guide tree construction: the guide tree is calculated from the distance matrix using the neighbor-joining algorithm[23]. The guide tree defines the order which the sequences are aligned in the next stage.

3. Progressive alignment: The sequences are progressively aligned in accordance to the guide tree.

Given $n$ number of sequences of length $m$, the distance matrix computation has a quadratic complexity of $O\left(n^{2} \mathrm{~m}^{2}\right)$. Profiling the three stages of ClustalW using gprof shows that the distance matrix computation is the most computationally intensive phase and typically takes up more than $90 \%$ of the overall runtime. Hence, it can be concluded that accelerating the distance matrix computation would provide a good speed up for the ClustalW.

Given a set of $n$ sequences $S=\left\{S_{1}, S_{2}, \ldots, S_{n}\right\}$. The distance of two sequences $S_{i}, S_{j}$ $\in S$, is defined by Equation (1).

$$
d\left(S_{i}, S_{j}\right)=1-\frac{\operatorname{nid}\left(S, S_{j}\right)}{\min \left\{l_{i}, l_{j}\right\}}
$$

where $\operatorname{nid}\left(S_{i}, S_{j}\right)$ denotes the number of exact matches in the optimal local alignment of $S_{i}$ and $S_{j}$ with respect to the given scoring system and $l_{i}$ and $l_{j}$ denotes the length of $S_{i}$ and $S_{j}$, respectively.

Liu et.al[14] states that given two sequences $S_{1}$ and $S_{2}$ with affine gap penalties $\alpha$ and $\beta$ and the substitution table $s b t$, a matrix $N_{A}(i, j)\left(1 \leq i \leq l_{l}, 1 \leq j \leq l_{2}\right)$ can be recursively defined as shown in Equation 2.

$$
\begin{aligned}
& N_{A}(i, j)= \\
& \left\{\begin{array}{cc}
0, & \text { if } H_{A}(i, j)=0 \\
N_{A}(i-1, j-1)+m(i, j), & \text { if } H_{A}(i, j)=H_{A}(i-1, j-1)+\operatorname{sbt}\left(S_{1}[i], S_{2}[j]\right) \\
N_{E}(i, j-1), & \text { if } H_{A}(i, j)=E(i, j) \\
N_{F}(i, j), & \text { if } H_{A}(i, j)=F(i, j)
\end{array}\right.
\end{aligned}
$$

where

$$
\begin{aligned}
& m(i, j)=\left\{\begin{array}{l}
1, \text { if } S_{1}[i]=S_{2}[j] \\
0, \text { otherwise }
\end{array}\right. \\
& N_{E}(i, j)=\left\{\begin{array}{l}
0, \\
N_{A}(i, j-1), \text { if } E(i, j)=H_{A}(i, j-1)-\alpha \\
N_{E}(i, j-1), \text { if } E(i, j)=E(i, j-1)-\beta
\end{array}\right.
\end{aligned}
$$




$$
\begin{aligned}
& N_{F}(i, j)=\left\{\begin{array}{l}
0, \quad \text { if } j=1 \\
N_{A}(i-1, j), \text { if } F(i, j)=H_{A}(i-1, j)-\alpha \\
N_{F}(i-1, j), \text { if } F(i, j)=F(i-1, j)-\beta
\end{array}\right. \\
& H_{A}=\max \left\{\begin{array}{l}
0, \\
E(i, j), \\
F(i, j), \\
H_{A}(i-1, j-1)+\operatorname{sbt}\left(S_{1}[i], S_{2}[j]\right)
\end{array}\right. \\
& E(i, j)=\max \left\{H_{A}(i, j-1)-\alpha, E(i, j-1)-\beta\right\} \\
& F(i, j)=\max \left\{H_{A}(i-1, j)-\alpha, F(i-1, j)-\beta\right\}
\end{aligned}
$$

Using the matrix $N_{A}(i, j)$, the distance value $d\left(S_{i}, S_{j}\right)$ can then be redefined as shown in Equation 3.

$$
d\left(S_{i}, S_{j}\right)=1-\frac{N A\left(i_{\max }, j_{\max }\right)}{\min \left\{l_{i}, l_{j}\right\}}
$$

A more detailed explanation and proof of these formulas is described in[14].

\section{Mapping onto the Cell BE Architecture}

Figure 2 illustrates the mapping of distance matrix computation onto the Cell BE.

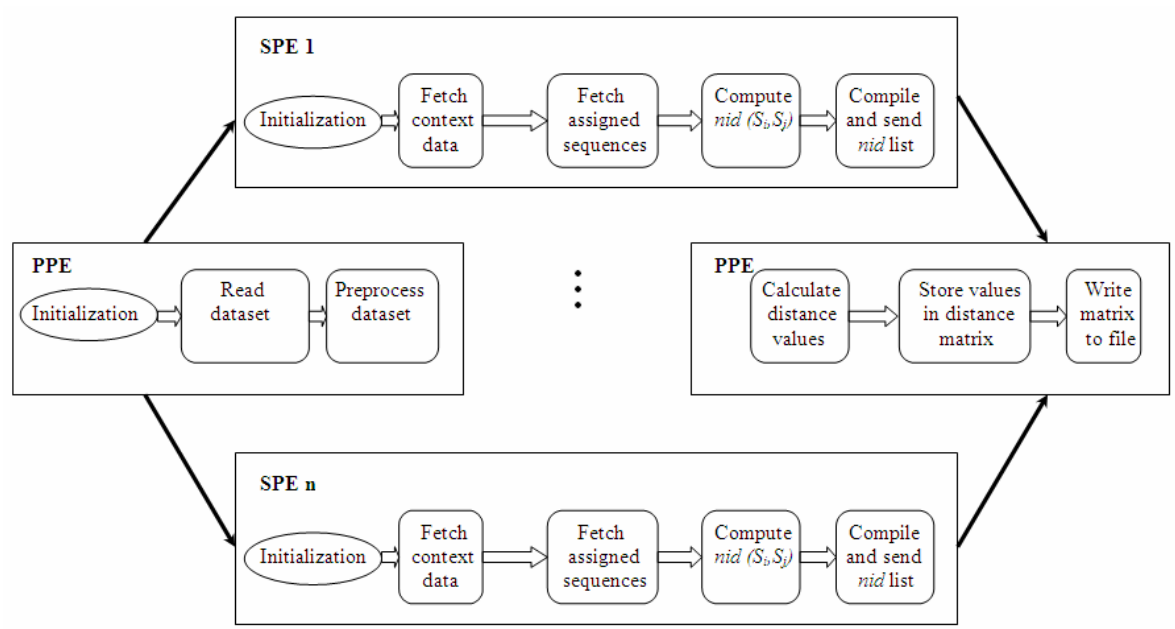

Fig. 2. Mapping of our distance matrix computation algorithm onto the Cell B.E. 
The algorithm starts by reading the input dataset. The PPE then preprocesses and divides the dataset into equal size blocks for each SPEs to process. Since the blocks are independent of each other, no thread synchronization is necessary during the calculations. The mailbox functions spe_in_mbox_write and spu_read_in_mbox are used to ensure that all the SPEs obtain their respective contexts in their local memory. Using the context data, each SPE then transfers any required information and necessary sequences. To improve transfer efficiency, the database sequences in main memory and in the local storage are aligned within the cache line and data structures are initialized during the transfer of the sequence. Once it has finished calculating all its respective $\operatorname{nid}\left(S_{i}, S_{j}\right)$ scores, each SPEs sends the scores to the PPE in the form of a list. The PPE compiles the lists, calculates the distance values, and stores them in the distance matrix. The matrix is then written to a text file. The SPE pseudocode is shown in Figure 3.

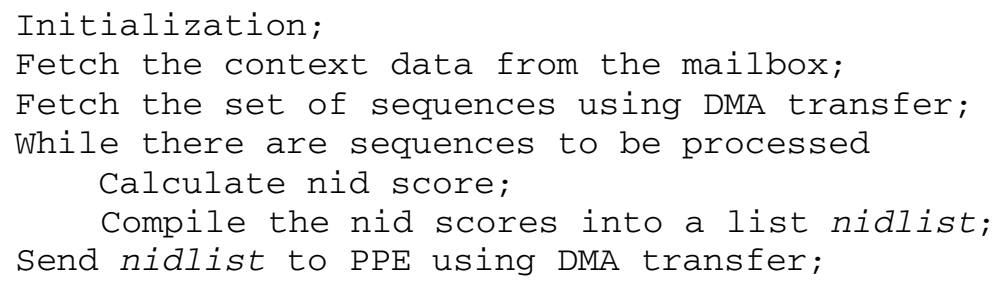

Fig. 3. Pseudocode of the SPE code

The $\operatorname{nid}\left(S_{i}, S_{j}\right)$ scores are computed in the SPEs in parallel using the Single Instruction Multiple Data (SIMD) registers using SPU intrinsics[24]. The pseudocode of the nid score calculation is shown in Figure 4.

Based on Equation (3), nid $\left(S_{i}, S_{j}\right)$ can be computed without computation of the actual traceback, which can cost a lot of resources from storing the complete dynamic programming matrix especially for long sequences. Since all elements in the same minor diagonal of the dynamic programming matrix can be computed independent of each other in parallel, the computation is done in minor diagonal order. Given are the vector registers $v H, v E, v F, v N_{A}, v N_{E}$ and $v N_{F}$ containing the values $H_{A}, E, F, N_{A}, N_{E}$ and $N_{F}$, respectively. For each loop of $c\left(1 \leq c \leq\left(l_{1}+l_{2}-1\right)\right)$, the values of $H_{A}, E, F, N_{A}$, $N_{E}$ and $N_{F}$ are computed. Calculations of the $v H, v E$, and $v F$ vectors are done by utilizing the spu_cmpgt intrinsic, which compares each element of a vector with the corresponding element of another vector, to create vector masks. The masks are then used as patterns to generate the resulting vector using the spu_sel intrinsic, which selects the corresponding bit from either vector in accordance to a provided pattern vector. The masks used in the $v E, v F$ and $v H$ computations are used to determine the value of the corresponding $v N_{E}, v N_{F}$ and $v N_{A}$ vectors, respectively. 


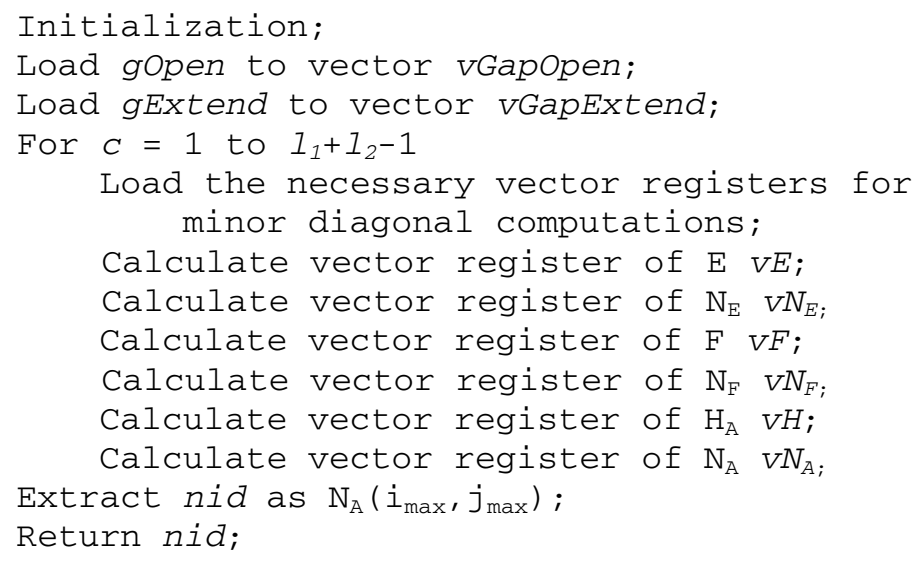

Fig. 4. Pseudocode of nid score calculation

\section{Performance Evaluation}

A set of experiments has been conducted using different numbers of protein sequences i.e. 400 sequences of average length 408,600 sequences of average length 462,800 sequences of average length 454 , and 1000 sequences of average length 446 . The measured runtimes are then compared to the original ClustalW implementation and the GPU-ClustalW implementation described in[14].

All experiments have been carried out on a standalone PS®3 with Fedora Core 9.0 operating system and the Cell Software Development Kit (SDK) 3.1. The sequential ClustalW application, available online at http://www.bii.a-star.edu.sg/achievements/ applications/clustalw/, was benchmarked on an Intel Pentium $43.0 \mathrm{GHz}$ processor with 1 GB RAM running on Windows XP. The GPU-ClustalW implementation was conducted with Nvidia GeForce 7900 GTX graphic card with a $717 \mathrm{MHz}$ engine clock speed, a $1.79 \mathrm{GHz}$ memory clock speed, 8 vertex processors, 24 fragment processors, and a $512 \mathrm{MB}$ memory, running on Pentium 43.0 GHz, 1 GB RAM with Windows XP.

Table 1 shows the performance evaluation of our parallel algorithm using the above mentioned datasets. The term $n(m)$ describes a dataset containing $n$ sequences

Table 1. Runtime comparison of distance matrix computation. The timing is measured in seconds.

\begin{tabular}{ccrrr}
\hline \#sequences & 400 & 600 & 800 & 1000 \\
(average length) & $(408)$ & \multicolumn{1}{c}{$462)$} & \multicolumn{1}{c}{$(454)$} & \multicolumn{1}{c}{$446)$} \\
\hline ClustalW & 833.1 & 1697.0 & 2966.6 & 4409.6 \\
GPU-ClustalW & 73.7 & 150.0 & 249.0 & 368.8 \\
Playstation ${ }^{\circledR} 3$ & 11.0 & 20.4 & 29.5 & 40.8 \\
\hline
\end{tabular}


with an average length of $m$. By using all 6 SPEs available on the Playstation 3 , our parallel algorithm achieves a runtime of 40.82 seconds for a dataset input of 1000 sequences of average length 446 .

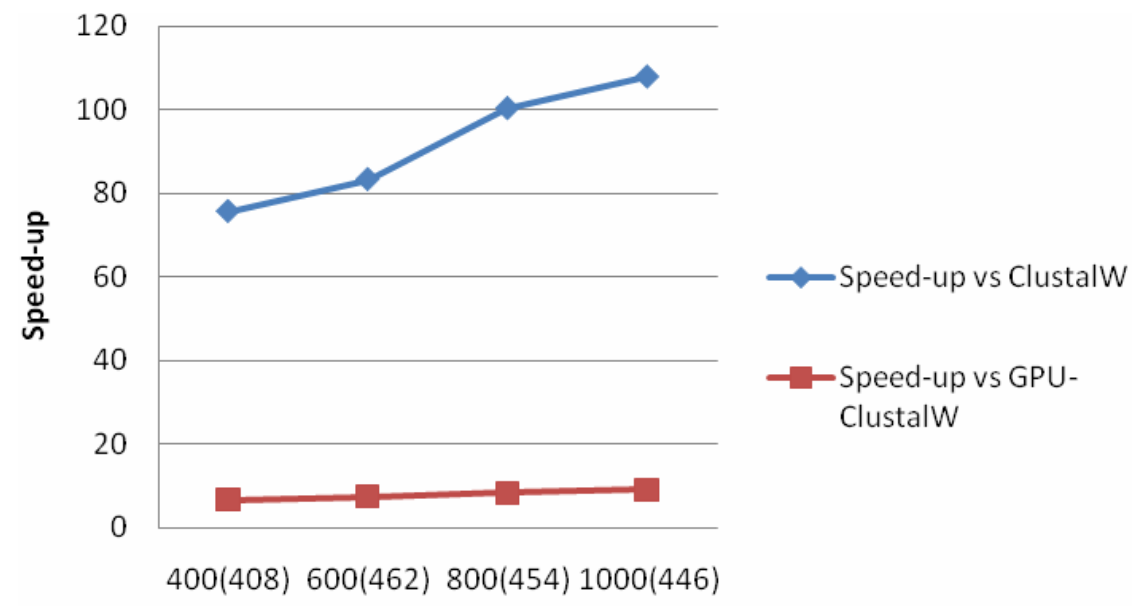

Number of sequences (average length)

Fig. 5. Speed-up comparison of our implementation on the Playstation ${ }^{\circledR} 3$ with ClustalW and GPU-ClustalW

Figure 5 shows the speed-up obtained by our implementation compared to the ClustalW and GPU-ClustalW. Our implementation obtains an average speed-up of 91.87x over all the datasets compared to the ClustalW implementation, with a peak speed-up of 108.03x for the 1000(446) dataset. The average speed-up of our implementation over the GPU-ClustalW is $7.87 \mathrm{x}$, with a peak speed up of $9.03 \mathrm{x}$ for the 1000(446) dataset.

Table 2. Detailed performance analysis of our parallel algorithm. The terms $T$ and $S$ describe the runtime and the speed-up compared to the previous row, respectively.

\begin{tabular}{|c|c|c|c|c|c|c|c|c|c|}
\hline \multirow[t]{2}{*}{$\begin{array}{c}\text { \#sequences } \\
\text { (average length) }\end{array}$} & \multirow[t]{2}{*}{ Processor } & \multicolumn{2}{|c|}{$\begin{array}{c}400 \\
(408)\end{array}$} & \multicolumn{2}{|c|}{$\begin{array}{c}600 \\
(462)\end{array}$} & \multicolumn{2}{|c|}{$\begin{array}{c}800 \\
(454)\end{array}$} & \multicolumn{2}{|c|}{$\begin{array}{l}1000 \\
(446)\end{array}$} \\
\hline & & $\mathrm{T}$ & $\mathrm{S}$ & $\mathrm{T}$ & $\mathrm{S}$ & $\mathrm{T}$ & $\mathrm{S}$ & $\mathrm{T}$ & $\mathrm{S}$ \\
\hline $\begin{array}{l}\text { Baseline } \\
\text { ClustalW }\end{array}$ & $\begin{array}{c}\text { Pentium } 4 \\
3.0 \mathrm{GHz}\end{array}$ & 833.1 & N.A & 1697.0 & N.A & 2966.6 & N.A & 4409.6 & N.A \\
\hline $\begin{array}{l}\text { Baseline } \\
\text { ClustalW }\end{array}$ & PPE & 667.86 & 1.24 & 1361.13 & 1.24 & 2379.0 & 1.24 & 3536.2 & 1.24 \\
\hline $\begin{array}{l}\text { Non-vectorized } \\
\text { code }\end{array}$ & PPE & 357.89 & 1.87 & 717.83 & 1.89 & 1702.08 & 1.80 & 1871.08 & 1.89 \\
\hline Vectorized code & PPE+1SPE & 57.15 & 6.26 & 113.41 & 6.33 & 168.54 & 7.83 & 237.12 & 7.89 \\
\hline Vectorized code & PPE+6SPEs & 11.01 & 5.19 & 20.36 & 5.57 & 29.53 & 5.71 & 40.82 & 5.81 \\
\hline
\end{tabular}


Table 2 shows a more detailed performance analysis of our parallel algorithm using the above mentioned datasets. It compares the runtimes of our implementation and the baseline ClustalW on various processors. The performance analysis breaks down the speedup obtained by each phase of the improvement made by our implementation. The non-vectorized code is implemented according to the algorithm described in section 3, without the use of SIMD vectorization. The vectorized code is implemented according to section 4 .

\section{Conclusion}

We have presented a parallel algorithm on the Cell B.E. heterogeneous multi-core system for the distance matrix computation used in multiple sequence alignment algorithms. Our implementation on the Playstation $₫ 3$ achieves an average speed-up of $91.87 x$ compared to the publicly available sequential ClustalW implementation.

\section{References}

1. Lipman, D.J., Altschul, S.F., Kececioglu, J.D.: A tool for multiple sequence alignment. Proceedings of the National Academy of Sciences of the United States of America 86(12), 4412-4415 (1989)

2. Thompson, J.D., Higgins, D.G., Gibson, T.J.: CLUSTAL W: improving the sensitivity of progressive multiple sequence alignment through sequence weighting, position-specific gap penalties and weight matrix choice. Nucl. Acids Res. 22(22), 4673-4680 (1994)

3. Notredame, C., Higgins, D.G., Heringa, J.: T-coffee: A novel method for fast and accurate multiple sequence alignment. Journal of Molecular Biology 302(1), 205-217 (2000)

4. Katoh, K., Misawa, K., Kuma, K.I., Miyata, T.: MAFFT: A novel method for rapid multiple sequence alignment based on fast Fourier transform. Nucleic Acids Research 30(14), 3059-3066 (2002)

5. Schmollinger, M., Nieselt, K., Kaufmann, M., Morgenstern, B.: DIALIGN P: Fast pairwise and multiple sequence alignment using parallel processors. BMC Bioinformatics, 5 (2004)

6. Simossis, V.A., Heringa, J.: PRALINE: A multiple sequence alignment toolbox that integrates homology-extended and secondary structure information. Nucleic Acids Research 33(suppl. 2), W289-W294 (2005)

7. Thompson, J.D., Gibson, T.J., Plewniak, F., Jeanmougin, F., Higgins, D.G.: The CLUSTAL_X windows interface: flexible strategies for multiple sequence alignment aided by quality analysis tools. Nucl. Acids Res. 25(24), 4876-4882 (1997)

8. Catalyurek, U., Stahlberg, E., Ferreira, R., Saltzt, J.: Improving Performance of Multiple Sequence Alignment Analysis in Multi-client Environments. In: Proceedings of the First International Workshop on High Performance Computational Biology 2002 (HiCOMB 2002, IPDPS 2002) (2002)

9. Catalyurek, U., Gray, M., Kurc, T., Saltzt, J., Stahlberg, E., Ferreira, R.: A componentbased implementation of multiple sequence alignment. In: Proceedings of the ACM Symposium on Applied Computing: 2003, pp. 122-126 (2003)

10. Li, K.-B.: ClustalW-MPI: ClustalW analysis using distributed and parallel computing. Bioinformatics 19(12), 1585-1586 (2003) 
11. Chaichoompu, K., Kittitornkun, S., Tongsima, S.: MT-ClustalW: Multithreading multiple sequence alignment. In: 20th International Parallel and Distributed Processing Symposium, IPDPS 2006 (2006)

12. Luo, J., Ahmad, I., Ahmed, M., Paul, R.: Parallel multiple sequence alignment with dynamic scheduling. In: International Conference on Information Technology: Coding and Computing, ITCC 2005, pp. 8-13 (2005)

13. Oliver, T., Schmidt, B., Nathan, D., Clemens, R., Maskell, D.: Multiple sequence alignment on an FPGA. In: Proceedings of the International Conference on Parallel and Distributed Systems - ICPADS 2005, pp. 326-330 (2005)

14. Liu, W., Schmidt, B., Voss, G., Muller-Wittig, W.: Streaming Algorithms for Biological Sequence Alignment on GPUs. IEEE Transactions on Parallel and Distributed Systems (2007)

15. Kahle, J.A., Day, M.N., Hofstee, H.P., Johns, C.R., Maeurer, T.R., Shippy, D.: Introduction to the Cell multiprocessor. IBM Journal of Research and Development 49(45), 589-604 (2005)

16. Pande, V.: Folding@ Home: Using Worldwide distributed computing to break fundamental barriers in molecular simulation. In: Proceedings of the IEEE International Symposium on High Performance Distributed Computing 2006, p. 4 (2006)

17. Sachdeva, V., Kistler, M., Speight, E., Tzeng, T.-H.K.: Exploring the viability of the Cell Broadband Engine for bioinformatics applications. In: IEEE International Parallel and Distributed Processing Symposium 2007, 8 p. IEEE, Long Beach (2007)

18. Wirawan, A., Kwoh, C.K., Hieu, N.T., Schmidt, B.: CBESW: Sequence alignment on the playstation 3. BMC Bioinformatics, 9 (2008)

19. Stamatakis, A., Ludwig, T., Meier, H.: RAxML-II: A program for sequential, parallel and distributed inference of large phylogenetic trees. Concurrency Computation Practice and Experience 17(14), 1705-1723 (2005)

20. Pham, D., Behnen, E., Bolliger, M., Hofstee, H.P., Johns, C., Kahle, J., Kameyama, A., Keaty, J., Le, B., Masubuchi, Y., et al.: The design methodology and implementation of a first-generation CELL processor: a multi-core SoC. In: Proceedings of the IEEE 2005 Custom Integrated Circuits Conference 2005, San Jose, CA, USA, pp. 45-49. IEEE, Los Alamitos (2005)

21. International Business Machines: Software Development Kit 2.1 Accelerated Library Framework Programmer's Guide and API Reference, Version 1.1. In: IBM developerWorks (2007)

22. Feng, D.F., Doolittle, R.F.: Progressive sequence alignment as a prerequisitetto correct phylogenetic trees. Journal of Molecular Evolution 25(4), 351-360 (1987)

23. Saitou, N., Nei, M.: The neighbor-joining method: a new method for reconstructing phylogenetic trees. Molecular biology and evolution 4(4), 406-425 (1987)

24. IBM: $\mathrm{C} / \mathrm{C}++$ Language Extensions for Cell Broadband Engine Architecture v.2.5. In. IBM developerWorks (2008) 